\title{
Conchas marinas: exquisitos termómetros
}

\author{
Javier Medina-Sánchez
}

\section{Resumen}

Casi todos hemos admirado la exquisita arquitectura de las conchas marinas, sus colores y disfrutado su delicioso contenido ¿Sabía usted que su química nos puede decir la temperatura del mar? Si tomamos la concha de una almeja o un ostión y analizamos las diferencias entre las masas de la multitud de átomos de oxígeno y carbono que la componen, podemos saber la temperatura a la que se formó, y con ello, la temperatura del agua. Esto es gracias a la novedosa técnica de isótopos estables agregados, una celebridad científica relativamente nueva y conocida internacionalmente por su nombre en inglés como "clumped isotopes". El descubrimiento de la propiedad que tienen algunos átomos pesados de unirse entre ellos con mayor o menor frecuencia, dependiendo de la temperatura, y la posibilidad de medir esta propiedad, abren grandes perspectivas de avances en numerosos campos científicos.

Palabras clave: isótopos estables, minerales biológicos, carbonatos de calcio, termómetro marino.

\section{SEASHELLS: EXQUISITE THERMOMETERS}

\begin{abstract}
Seashells are highly prized for their exquisite architecture, colours and in some cases, their delicious content. Did you know that the chemistry of those shells can tell us the temperature of the sea? If we analyse how common it is for oxygen and carbon atoms of different masses to come together within an oyster or mussel shell, we can work out the temperature at which those shells were formed, and from that we can infer the sea water temperature. This is possible thanks to the cutting-edge technique of clumped isotopes, one of the most recent international celebrities in Earth Sciences. The discovery many decades ago of the property of some heavy atoms to bond with each other more or less frequently, depending on the temperature, and the recent advances which have made it possible to measure such a property, have opened up new possibilities for diverse scientific fields.
\end{abstract}

Keywords: clumped stable isotopes, biominerals, calcium carbonate, thermometry

Recepción: 08/05/2020. Aprobación: 18/01/2021. Dol: http://doi.org/10.22201/cuaieed.16076079e.2021.22.2.1 
Graduado de la licenciatura en Biología por la unam y de la maestría en Ciencias en el Instituto de Ecología de la misma universidad. Doctor en Ciencias por la Universidad de Leicester en el Reino Unido. Sus investigaciones incluyen aspectos sobre ecología de comunidades en zonas áridas, geología y geomorfología del centro-sur de México. En el campo de los isótopos estables, ha llevado a cabo trabajos sobre cambio ambiental en el sur de África, cactus gigantes en México, así como paleotermometría con isótopos estables pesados en biominerales marinos en ambientes tropicales, meridionales y árticos. Actualmente se desempeña como miembro investigador (Research Fellow) en la Universidad de Stirling en el Reino Unido.

\section{Introducción}

Conocer cómo ha cambiado la temperatura del mar durante cientos, miles y millones de años ha sido un enorme reto para la comunidad científica dedicada a estudiar la evolución biológica y geológica en la Tierra. Este conocimiento es importante ya que permite tener ideas más claras sobre el futuro de los ecosistemas ante los cambios climáticos que se avecinan. Uno de los avances más importantes dentro de los estudios sobre el cambio climático pasado es el uso de moléculas formadas por diferentes combinaciones de carbono y oxígeno como termómetros (Eiler, 2007).

Las conchas marinas están hechas de una sustancia llamada carbonato de calcio, y cada una de estas moléculas está formada de un átomo de calcio (Ca), uno de carbono $(\mathrm{C})$ y tres de oxígeno $\left(\mathrm{O}_{3}\right)$. Su fórmula química se expresa como $\mathrm{CaCO}_{3}$. Este es un mineral blanquecino al que las conchas le deben su dureza y durabilidad; mientras que su gran variedad de colores se la deben a otros compuestos orgánicos. Lo que crea una exquisita diversidad (Ver la Figura 1). 
Figura 1. Los biominerales marinos, con su exquisita arquitectura y colores, son además una rica fuente de información científica.

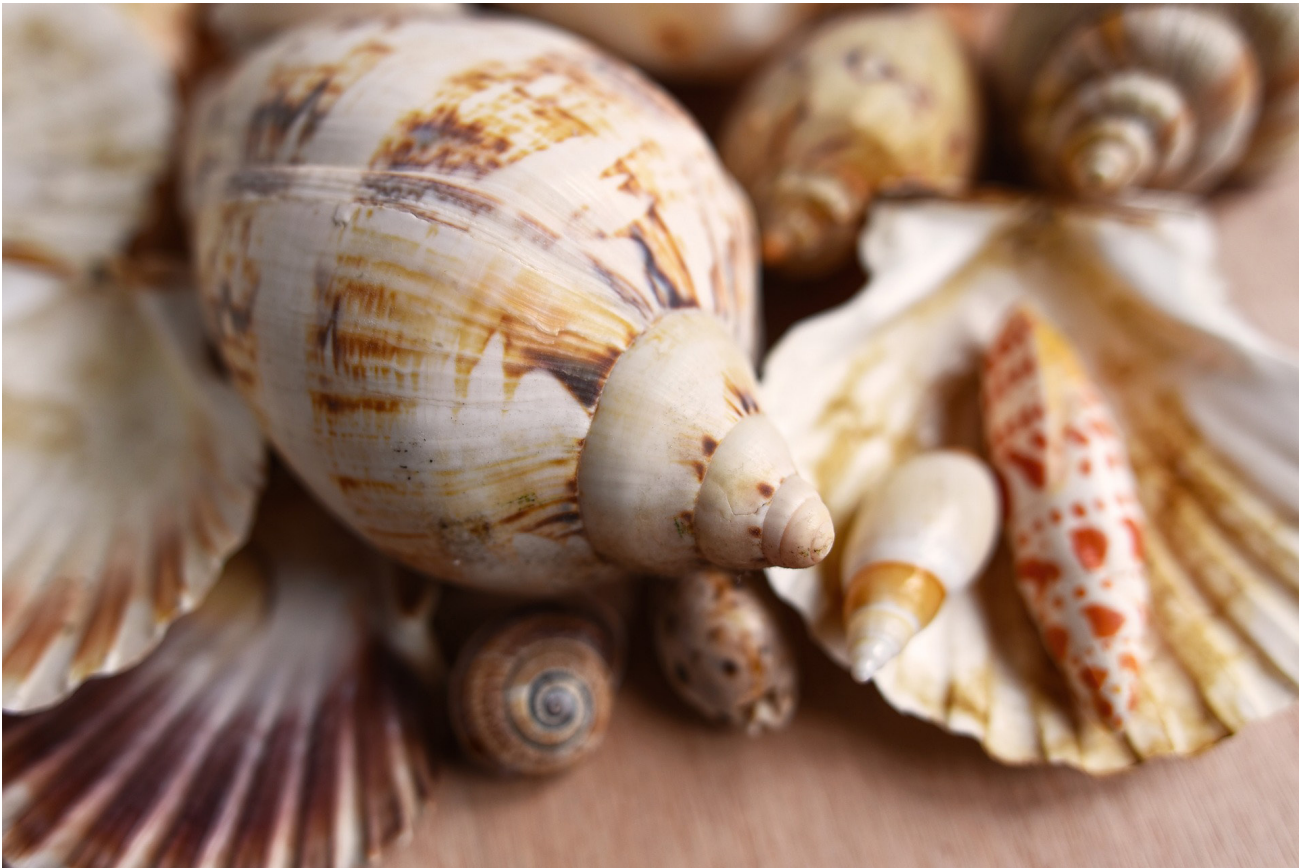

Debido a que el carbonato de calcio es un mineral hecho por un ser vivo, le Ilamamos biomineral (Crichton, 2019). La parte dura de nuestros dientes es también un ejemplo de biomineral, pero hecho en su mayor parte de fosfato de calcio. Sorprendentemente, muchos biominerales de carbonato de calcio representan una valiosa fuente de información sobre la temperatura del mar en el pasado geológico. A continuación, se presenta una breve explicación sobre cómo es que este biomineral puede funcionar como termómetro.

\section{Un mar de termómetros}

Para crear sus conchas de carbonato de calcio, los organismos marinos toman los elementos químicos del agua que les rodea, y al hacerlo llevan a su interior información de la temperatura del momento. Además de los moluscos, existen muchos otros organismos marinos que crean estructuras duras en forma de conchas y esqueletos de carbonato de calcio, como por ejemplo, los corales, algunas algas, los braquiópodos, las galletas y el erizos de mar; también algunos organismos microscópicos como los foraminíferos. Estos seres vivos han depositado sus conchas y esqueletos en el fondo del mar de manera continua, durante muchos millones de años, incluso formando muchas de las rocas que hoy en día se observa en la corteza terrestre. Debido a esto podemos decir que los biominerales de carbonato de calcio marino son, en conjunto, uno de los termómetros más abundantes en nuestro planeta, son un verdadero mar de termómetros. 


\section{El termómetro de los pesos pesados}

La clave, para investigar la temperatura del agua a la que los biominerales se formaron, está en el carbono y en el oxígeno de la molécula de carbonato de calcio. Primero hay que recordar que no todos los átomos de un elemento son iguales. Consideremos a el carbono como ejemplo, en la naturaleza algunos átomos de carbono tienen una mayor masa que otros, el peso pesado más conocido es el carbono catorce, identificado con el símbolo ${ }^{14} \mathrm{C}$ (el número 14 es el resultado de sumar los 6 protones y los 8 neutrones en su núcleo). Mucho se ha hablado del carbono catorce en disciplinas como la arqueología y en estudios del cambio climático por su uso para conocer la edad de algunas moléculas orgánicas antiguas, pero no es útil como termómetro debido a su propiedad radiactiva que provoca que cambie con el tiempo hasta convertirse en uno más ligero.

Existen otros átomos de carbono más ligeros que el ${ }^{14} \mathrm{C}$, que no son radioactivos y que por ello no cambian con el tiempo, el doce y el trece $\left({ }^{12} \mathrm{C} y{ }^{13} \mathrm{C}\right)$. A estos "hermanos" les llamamos isótopos estables, siendo el ${ }^{13} \mathrm{C}$ el más pesado de los dos por tener en su núcleo un neutrón más que el ${ }^{12} \mathrm{C}$. De la misma forma, existen oxígenos ligeros y pesados; pero para nuestro termómetro, sólo sirven el dieciséis $\left({ }^{16} \mathrm{O}\right)$, diecisiete $\left({ }^{17} \mathrm{O}\right)$ y dieciocho $\left({ }^{18} \mathrm{O}\right)$. Cuando tenemos dos o más isótopos pesados de estos elementos en la misma molécula de $\mathrm{CO}_{2}$, les llamamos isótopos agregados. Si le es más fácil para recordar, usted les puede llamar: agrupados, amontonados, aglomerados, en mazacote, apelotonados, en bola, congregados, etc. (Ver Figura 2).

Figura 2. Cuando en una molécula de dióxido de carbono hay dos o más isótopos pesados, se les considera como isótopos agregados. Fuente: elaboración propia.
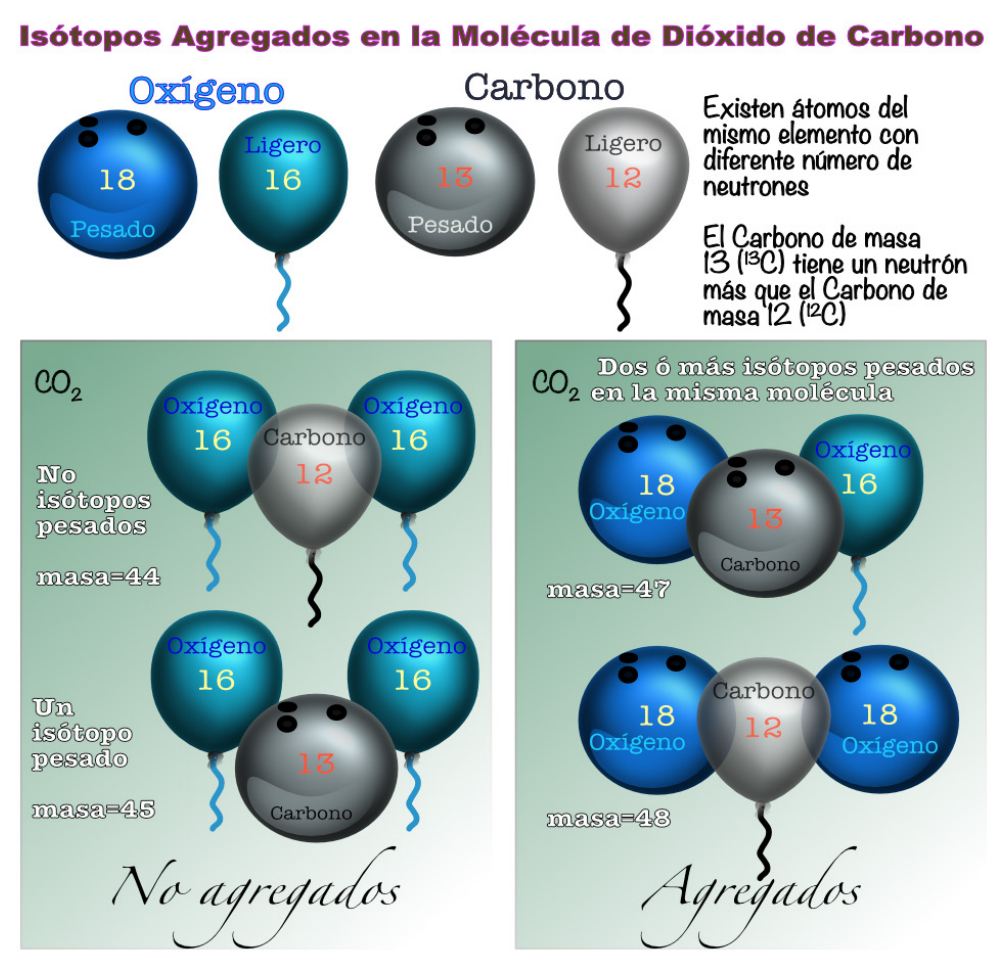
Son esas diferencias de peso las que se usan para saber la temperatura a la que se formó el biomineral, así, podemos encontrar moléculas de carbonato de calcio formadas exclusivamente de isótopos ligeros ( $\mathrm{Ca}^{12} \mathrm{C}{ }^{16} \mathrm{O}{ }^{16} \mathrm{O}{ }^{16} \mathrm{O}$ ); solamente de sus versiones más pesadas ( $\mathrm{Ca}{ }^{13} \mathrm{C}^{18} \mathrm{O}{ }^{18} \mathrm{O}{ }^{18} \mathrm{O}$ ) o combinaciones intermedias (Ca $\left.{ }^{12} \mathrm{C}^{17} \mathrm{O}{ }^{16} \mathrm{O}{ }^{17} \mathrm{O}\right)$. Para calcular la temperatura debemos medir qué proporción de todas las moléculas de carbonato de calcio de una muestra están formadas por dos o más átomos pesados. Otra diferencia fundamental en este termómetro es que los pesos pesados son extremadamente raros, en la naturaleza solo el 0.04 $\%$ del oxígeno es ${ }^{17} \mathrm{O}$ y el $0.2 \%$ es ${ }^{18} \mathrm{O}$, mientras que el restante $99.76 \%$ es ${ }^{16} \mathrm{O}$ (Vea el sitio web de la International Atomic Energy Agency).

\section{Arrímese, que hace frío}

Para formar sus biominerales de carbonato de calcio, los seres vivos tienen a su disposición una mezcla de isótopos de carbono y oxígeno ligeros contenidos en las moléculas de dióxido de carbono $\left(\mathrm{CO}_{2}\right)$, carbonatos $\left(\mathrm{CO}_{3}{ }^{2-}\right)$ y bicarbonatos $\left(\mathrm{HCO}_{3}{ }^{-}\right)$que están disueltos en el agua. Hay dos aspectos a considerar: primero, los átomos ligeros vibran y reaccionan más rápido que los pesados para unirse a otros y formar nuevas moléculas; segundo, al disminuir la temperatura, también lo hace la velocidad a la que todos los átomos reaccionan.

Para formar moléculas de carbonato de calcio, los átomos de calcio, carbono y oxígeno tienen que "colisionar" entre sí en una reacción química. Imaginemos que vamos a formar muchas millones de moléculas de $\mathrm{CaCO}_{3^{\prime}}$ con sólo el $0.2 \%$ de los átomos disponibles de cada elemento siendo pesados $\left({ }^{13} \mathrm{C}\right.$, ${ }^{18} \mathrm{O}$ y ${ }^{17} \mathrm{O}$ ) y el resto (99.8\%) siendo ligeros $\left({ }^{12} \mathrm{C} \mathrm{y}^{16} \mathrm{O}\right)$. Cuando hace calor, es como un gran baile en un verano tropical y con mucha "buena vibra", por lo que es más probable que, al "bailar" a mucha velocidad, cada uno de los pesos pesados se unan por separado a uno ligero, ya que las vibraciones y colisiones entre los átomos son rápidas y la mayor parte de ellos son ligeros. Así, la inmensa mayoría de las moléculas de carbonato de calcio estaría formada exclusivamente de isótopos ligeros ( $\left.\mathrm{Ca}^{12} \mathrm{C}{ }^{16} \mathrm{O}{ }^{16} \mathrm{O}{ }^{16} \mathrm{O}\right)$; una proporción muy pequeña tendría un solo isótopo pesado $\left(\mathrm{Ca}^{12} \mathrm{C}^{16} \mathrm{O}{ }^{16} \mathrm{O}{ }^{18} \mathrm{O}\right)$; mientras que en casos extremadamente raros encontraríamos dos o más isótopos pesados en la misma molécula de carbonato de calcio (por ejemplo: $\mathrm{Ca}^{13} \mathrm{C}^{16} \mathrm{O}{ }^{16} \mathrm{O}{ }^{18} \mathrm{O}$ ).

Al bajar la temperatura, los pesos pesados se unen entre sí con mayor frecuencia. Ahora imaginemos que llega la madrugada y hace frío, en el baile de isótopos, los más pesados se vuelven todavía más lentos y son los últimos en levantarse de su silla al iniciar la música; los isótopos ligeros forman pareja primero y los pesados se van quedando solos, por lo que su única opción es formar pareja con otros pesos pesados. Al bajar la temperatura, encontraremos más parejas formadas de dos pesados. Otra forma de entenderlo es pensar en un grupo de pingüinos emperador congregados durante una fuerte tormenta, los pingüinos pesados se mueven con mayor lentitud y por ello son los últimos en unirse al grupo. 
Figura 3. El termómetro de isótopos agregados solo sirve si al disminuir la temperatura, los isótopos pesados se agrupan con más frecuencia de lo que lo harían al azar. Las uniones al azar entre átomos pesados y ligeros ocurren a temperaturas

muy altas, cerca de los mil grados centígrados. Fuente: elaboración propia.
Así que, al bajar la temperatura del agua del mar, podemos encontrar con más frecuencia dos, o más isótopos pesados unidos entre sí formando la misma molécula de carbonato de calcio en una concha o esqueleto de un organismo marino (por ejemplo: $\mathrm{Ca}^{13} \mathrm{C}^{16} \mathrm{O}{ }^{16} \mathrm{O}{ }^{18} \mathrm{O}$ y $\mathrm{Ca}^{13} \mathrm{C}{ }^{16} \mathrm{O}{ }^{17} \mathrm{O}{ }^{18} \mathrm{O}$ ). En resumen, entre más fría sea el agua, mayor será la proporción de moléculas hechas de dos o más isótopos pesados en este biomineral (Ver Figura 3).

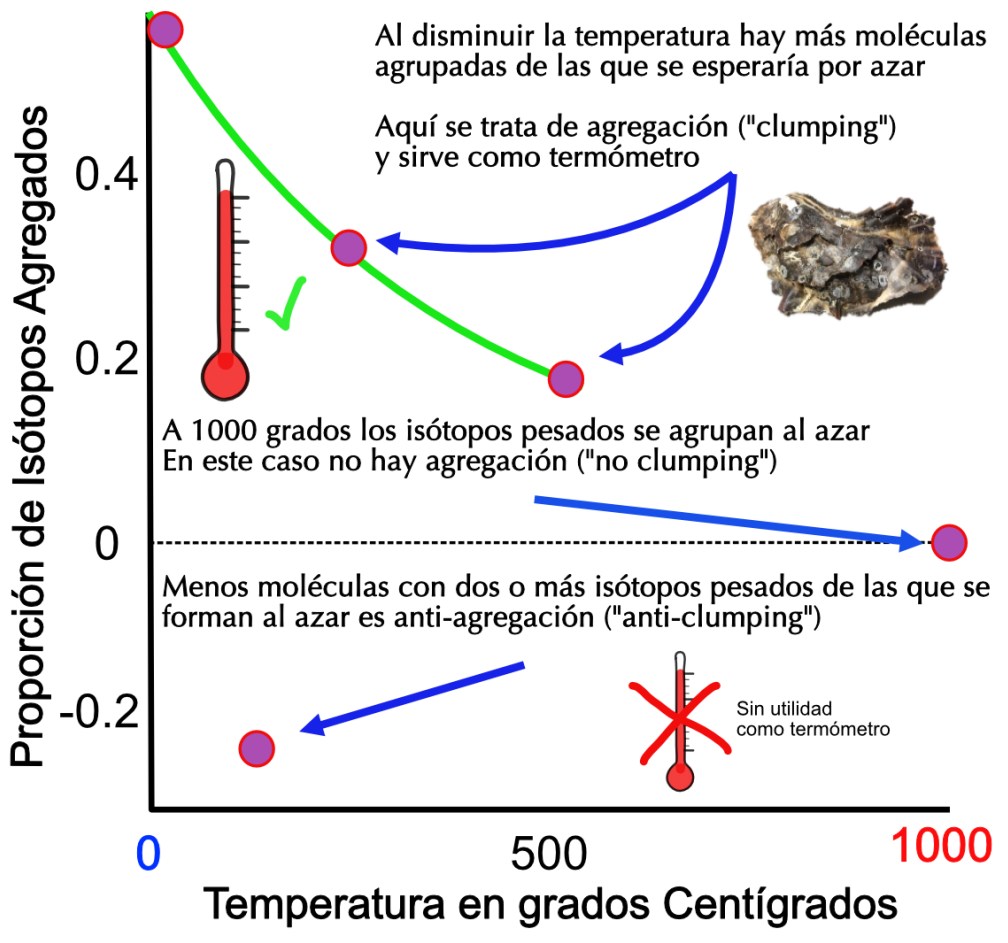

\section{¿Qué tan caliente es frío?}

Para calibrar el termómetro de isótopos agrupados, primero hay que medir su proporción en una muestra y compararla con una ya conocida, llamada estándar que en este caso es el valor al azar. Funciona así: si calentamos una muestra de $\mathrm{CO}_{2}$ a mil grados o más, los átomos ligeros y pesados se unirán entre ellos al azar, dependiendo solamente del número de ligeros y pesados de cada elemento, no de su masa, porque debido a tanta energía disponible, el peso no hace ninguna diferencia y todos se comportan prácticamente igual, como si todos pesaran lo mismo; es decir, no hay agregación de isótopos. Dicho de otra forma, a mil grados, la agregación es igual a cero y a partir de ese punto, al bajar la temperatura, los pesados se agregaran con más frecuencia de lo que lo harían a 1000 grados (agregación >0), así que podemos concluir que estos no se agregan al azar (Ver Figura 3). 


\section{Una báscula para las moléculas}

Para saber las diferencias de masa del oxígeno y el carbono no se usa estrictamente una báscula, sino un instrumento llamado espectrómetro de masas de proporciones de isótopos. Lo primero que se hace para interrogar a los biominerales de carbonato de calcio es disolverlos en ácido muy caliente, generalmente a $90{ }^{\circ} \mathrm{C}$. Al hacerlo se obtiene el gas dióxido de carbono $\left(\mathrm{CO}_{2}\right)$, el cual se purifica y se hace pasar a lo largo de un tubo al vacío donde se le quita un electrón, provocando que se convierta en ion, es decir, adquiere una carga eléctrica.

Las moléculas continúan su trayectoria, llegando a una parte en forma de "L" del tubo al vacío, misma que está en contacto con un enorme imán que es el encargado de desviar la trayectoria de las moléculas cargadas eléctricamente dependiendo de su peso. Las moléculas de $\mathrm{CO}_{2}$ más pesadas (mazacotes de isótopos) sólo chocarán con el detector que esté calibrado, alineado y posicionado para recibirles al final del tubo; mientras que las moléculas de menor masa (hechas de isótopos ligeros) adoptan trayectorias diferentes y llegan a otros detectores. De esta forma podemos medir la proporción de isótopos estables agregados en una muestra.

\section{Un termómetro en peligro}

La evolución ha permitido el desarrollo de múltiples formas de biominerales. La biología de cada especie es la encargada de controlar de manera exquisita y con mucho rigor tanto el tipo de mineral que se produce, como la forma en que se une cada uno de los miles de minerales individuales para formar estructuras como las conchas. Dependiendo de la forma en que los átomos de $\mathrm{CaCO}_{3}$ se distribuyen en el espacio, se pueden formar diferentes tipos de mineral, como el aragonita, la calcita o el simple carbonato de calcio sin forma definida (amorfo). Pensemos en cada mineral como un ladrillo y cada especie biológica como un constructor que decide el tamaño, la forma, el tipo de material del que fabricará esos ladrillos y también decidirá la forma en que los unirá entre sí para construir una casa o edificio. Cada especie tiene la habilidad de controlar la fabricación de sus estructuras biominerales, y de ella depende la eficiencia de la función de cada biomineral.

La formación y estructura de los biominerales marinos pueden estar en riesgo debido al acelerado incremento en la emisión de $\mathrm{CO}_{2}$ a la atmósfera, producto de múltiples actividades humanas. Este gas, además de que contribuye enormemente al calentamiento global, tiene un gran efecto en la acidificación del mar, lo cual causa que, algunas especies como los mejillones, tengan un menor control sobre el tipo de biomineral que producen y sobre la forma en que se unen entre ellos. Así que la acidificación del mar puede afectar negativamente la función protectora de la concha de estos organismos (Fitzer, et al., 2016). La información sobre la temperatura del mar contenida en las conchas marinas, está en su química, y no en su estructura, por lo que los estudios de isótopos estables son de gran valor para entender futuros riesgos del cambio climático. 


\section{Usos de la termometría de isótopos estables agregados}

Los isótopos agrupados, además de saber la temperatura del mar, ya sea de hoy, o de millones de años atrás, pueden servir para hacer muchos otros descubrimientos. Por ejemplo, la técnica de termometría de isótopos pesados se emplea para conocer la temperatura corporal de algunos dinosaurios (Dawson et al., 2020); la velocidad a la que sistemas montañosos se han elevado (Spencer y Kim, 2015); así como para realizar estudios sobre el origen del metano, otro importante gas de efecto invernadero. Estas aplicaciones y muchas otras más son posibles gracias a que los carbonatos de calcio se forman en una multitud de sistemas terrestres, incluyendo sistemas no marinos, como suelos, lagos, cuevas y algunas plantas.

\section{Estudios de isótopos estables agregados en México}

El autor de este artículo, en colaboración con investigadores de la unAm en México y las Universidades de Stirling y Glasgow en el Reino Unido realizan investigaciones empleando isótopos agregados de la región de Tehuacán en el centro-sur de México donde estudian, con dicha técnica, muestras de moluscos fósiles para conocer la temperatura del agua del mar Cretácico hace más de 100 millones de años. También se analizan algas coralinas modernas de las costas de Australia, Costa Rica, Escocia, Groenlandia, del Pacífico Mexicano y Noruega, con el objetivo de refinar la calibración de nuestro exquisito termómetro y evaluar su aplicación usando otros grupos de organismos marinos productores de biominerales.

Otras interesantes líneas de investigación que se llevan a cabo actualmente en la reserva de la Biósfera de Tehuacán-Cuicatlán incluyen el estudio de biominerales formados por cactus gigantes, minerales de carbonatos en suelos modernos, en suelos fósiles y en rocas para obtener información sobre el levantamiento de una parte de la Sierra Madre del Sur. Estos estudios permitirán complementar trabajos previos sobre la evolución natural de la región. Se recomienda ver el video que el autor en colaboración con otros investigadores realizaron sobre el tema de termometría de isótopos pesados: https://vimeo. com/448922079.

\section{Conclusión}

El uso de los isótopos estables agrupados en fósiles hechos de carbonatos está revolucionando los estudios sobre el cambio climático, se trata de una técnica relativamente nueva, nacida de la Geoquímica y con apenas un poco más de una década de consolidación. Y aunque en el presente, solo existe un puñado de laboratorios en el mundo equipados para realizar tales trabajos, los avances recientes abren grandes perspectivas de descubrimientos en otros campos de la ciencia. 
Es tiempo de pensar qué estudios sobre procesos geológicos, químicos y biológicos pueden beneficiarse de la posibilidad de poner en la báscula a los pesos pesados de los isótopos y qué preguntas científicas relevantes se pueden responder y explorar si dichos avances analíticos pueden ayudar a resolver algunos de los problemas globales que enfrentamos todos.

\section{Referencias}

* Crichton, R. (2019). Biomineralization. [R. Crichton, ed.]. Biological Inorganic Chemistry, 3, 517-544.

Dawson, R. R., Field, D. J., Hull, P. M., Zelenitsky, D. K., Therrien, F. y Affek, H. P. (2020). Eggshell geochemistry reveals ancestral metabolic thermoregulation in Dinosauria. Science Advances, 6 (7), eaax9361. https://doi.org/10.1126/sciadv. aax9361

*iler, J. M. (2007). Clumped-isotope geochemistry-The study of naturallyoccurring, multiply-substituted isotopologues. Earth and Planetary Science Letters, 262, 309-327. https://doi.org/10.1016/j.epsl.2007.08.020

* Fitzer, S. C., Chung, P., Maccherozzi, F., Dhesi, S., Kamenos, N., Phoenix, V.R. y Cusack, M. (2016). Biomineral shell formation under ocean acidification: a shift from order to chaos. Scientific Reports, 6 (21076), 1-7. https://doi.org/10.1038/ srep21076

* Spencer, C. y Kim, S. (2015). Carbonate clumped isotope paleothermometry: a review of recent advances in $\mathrm{CO} 2$ gas evolution, purification, measurement and standardization techniques. Geosciences Journal, 19, 357-374. https://doi. org/10.1007/s12303-015-0018-1

\section{Sitios web}

- International Atomic Energy Agency: https://nds.iaea.org/relnsd/vcharthtml/ VChartHTML.html,.

\section{Cómo CITAR ESTE ARTículo}

* Medina-Sánchez, Javier. (2021, marzo-abril). Conchas marinas: exquisitos termómetros. Revista Digital Universitaria (RDU), 22(2). Dol: http://doi.org/10.22201/ cuaieed.16076079e.2021.22.2.1 